\title{
An Alternative Way to Reach the Ventricular Surface of the Sinuses of Valsalva: Antegrade Transseptal Approach
}

\author{
Serkan Cay ${ }^{1}$, Ozcan Ozeke ${ }^{1}$, Firat Ozcan ${ }^{1}$, Serkan Topaloglu ${ }^{1}$, and Dursun Aras $^{1}$ \\ ${ }^{1}$ University of Health Sciences, Ankara City Hospital
}

May 6, 2020

\begin{abstract}
None

Letter to the Editor

An Alternative Way to Reach the Ventricular Surface of the Sinuses of Valsalva: Antegrade Transseptal Approach
\end{abstract}

Serkan Cay, MD; Ozcan Ozeke, MD; Firat Ozcan, MD; Serkan Topaloglu, MD; Dursun Aras, MD

Department of Cardiology, Division of Arrhythmia and Electrophysiology, University of Health

Sciences, Ankara City Hospital, Ankara, Turkey

Correspondence

Serkan Cay, MD

Department of Cardiology,

Division of Arrhythmia and Electrophysiology,

University of Health Sciences, Ankara City Hospital,

Bilkent, 06800 Cankaya, Ankara, Turkey

E-mail: cayserkan@yahoo.com

To the Editor,

We have read with great interest the article entitled 'Left Sinus of Valsalva - Electroanatomic Basis and Outcomes with Ablation for Outflow Tract Arrhythmias' by Kapa et $\mathrm{al}^{1}$ in the latest issue of the journal. We would like to thank the authors for conceptualizing an idea about the common ablation point for outflow tract ventricular arrhythmias with different ECG characteristics, suggesting different origins. In the current study, operators reached the area below the left sinus of Valsalva using the catheter inversion technique via the retrograde transaortic route. In some cases, ablation attempts cannot be successful with this approach and even with the use of a long sheath although effective in most patients. In addition, the operator cannot reach the aortic route because of anatomic obstacles in the arterial system occasionally. Catheter manipulation, contact and the stability might be challenging using the catheter inversion technique due to various anatomical reasons including highly mobile aortic valve leaflets and increased dimensions of the aortic root and the left ventricle in some cases. 
The antegrade transseptal approach is routinely used to reach the infravalvular region in some centers. There are some fine details with the antegrade transseptal approach using a 'reverse S' shape of the mapping catheter. To reach the ventricular surface of anteriorly and superiorly located aortic cusps more comfortably, one should be performed the transseptal puncture from the anterior and inferior region of the interatrial septum, posterior to the non-coronary cusp using either a fluoroscopic marker (angiography or mapping catheter) or imaging tool (intracardiac or transesophageal echo). With the help of the long sheath (SL1, Abbott or Preface@, Biosense Webster), the mapping catheter can form a 'reverse S' curve and clockwise and counter-clockwise movements rotate the catheter toward the left and right cusps, respectively,

The figure demonstrates a case with premature ventricular complexes originating from below the right coronary cusp and mapping and ablation using the antegrade transseptal approach (Supplementary Videos).

Keywords: cusp; reverse S; transaortic; transseptal

\section{Funding}

None.

\section{Conflict of interests}

The authors declare that there are no conflict of interests.

\section{References}

1 - Kapa S, Mehra N, Deshmukh AJ, Friedman PA, Asirvatham SJ. Left sinus of Valsalva-Electroanatomic basis and outcomes with ablation for outflow tract arrhythmias. J Cardiovasc Electrophysiol . 2020;31(4):952959.

\section{Figure and Video legends}

Figure : Images were formed by merging of a 3D-Electroanatomic mapping system (EnSite Precision, Abbott) and the fluoroscopy. The mapping catheter reaches the ventricular surface of the right coronary cusp using the 'reverse S' curve via the antegrade transseptal approach in the right (A) and left (B) anterior oblique views. In panels $\mathrm{A}$ and $\mathrm{B}$, only the aortic root with 3 cusps and the ascending aorta were merged. In panels $\mathrm{C}$ and $\mathrm{D}$, the left ventricular cavity was shown with the earliest ventricular activation (dots) in the same region in the right $(\mathrm{C})$ and left $(\mathrm{D})$ anterior oblique views. In panels $\mathrm{E}$ and $\mathrm{F}$, combined 3DElectroanatomic mapping (the aorta and the left ventricle) was merged with the fluoroscopic image of the mapping catheter in the right $(\mathrm{E})$ and left $(\mathrm{F})$ anterior oblique views.

Video : Six videos (A-F) corresponding to 6 panels in the figure. 


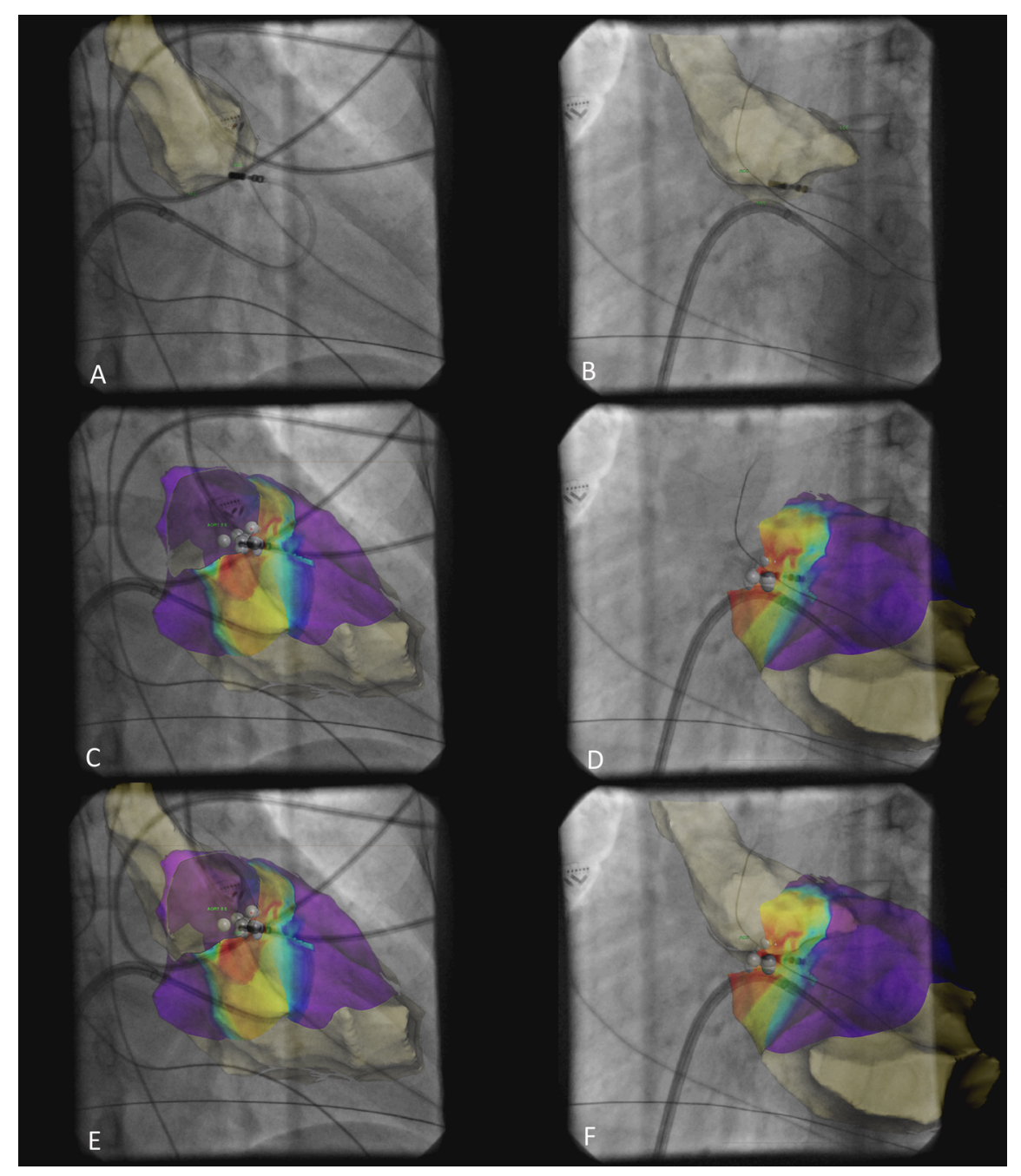

